\title{
Archéopages
}

Archéopages

Archéologie et société

Hors-série 3 | 2012

Nouveaux champs de la recherche archéologique

\section{Produire des connaissances en archéologie}

\section{Philippe Soulier}

\section{OpenEdition}

Journals

Édition électronique

URL : https://journals.openedition.org/archeopages/444

DOI : 10.4000/archeopages.444

ISSN : 2269-9872

\section{Éditeur}

INRAP - Institut national de recherches archéologiques préventives

\section{Édition imprimée}

Date de publication : 1 janvier 2012

Pagination : 16-20

ISSN : 1622-8545

\section{Référence électronique}

Philippe Soulier, «Produire des connaissances en archéologie », Archéopages [En ligne], Hors-série 3 |

2012, mis en ligne le 01 janvier 2012, consulté le 02 mars 2023. URL : http://journals.openedition.org/ archeopages/444 ; DOI : https://doi.org/10.4000/archeopages.444 


\section{Produire des connaissances en archéologie}

Philippe Soulier

CNRS, UMR 7041 "Archéologies et Sciences de l'Antiquité»

D epuis des décennies, les modalités de la recherche archéologique évoluent, que ce soit dans les pratiques de terrain, la professionnalisation des personnels de la recherche, la réglementation ou les moyens mis en œuvre. Cette évolution contribue directement à un accroissement massif des connaissances et pas une année ne se passe sans que des découvertes majeures ne viennent enrichir ce que l'on sait des sociétés du passé. Il s'agit maintenant, au-delà de la production et de la conservation, de savoir gérer et transmettre ces flux continus de données pour qu'elles profitent à tous en termes de connaissances. Mais la quantité des données et connaissances, anciennes et nouvelles, oblige, encore plus qu'avant, à une organisation rigoureuse qui en assure à la fois la validité, la protection et la traçabilité, et à une approche critique qui en caractérise la valeur intrinsèque et la représentativité extrinsèque.

Quelques rappels préliminaires. Cependant, avant de rentrer dans le vif du sujet, il est utile de rappeler quelques indications très générales, sur la production des connaissances en archéologie.

Aujourd'hui, l'«archéologie» est la discipline scientifique et patrimoniale qui étudie le passé des hommes à travers les traces et l'équipement matériels qui résultent de leurs activités, des origines de l'humanité aux périodes les plus récentes, des artefacts et écofacts les plus tenus aux paysages façonnés.

La «fouille archéologique» est l'un des principaux moyens d'accéder à ces traces et vestiges résiduels lorsqu'ils sont enfouis. Son caractère unique et rédhibitoire, car destructeur de son propre objet, impose des responsabilités particulières à l'archéologue fouilleur. En effet, les vestiges et sédiments collectés, les enregistrements des vestiges et des traces exhumés, ainsi que les notes de fouille qui leur donnent sens, constituent tout ce qui restera du site. Ce sont ces données primaires qui forment le noyau dur des futures connaissances.

À chaque opération de fouille correspond un rapport, quelle qu'en soit la formalisation. Celui-ci est le lieu de la présentation des premiers résultats, argumentés par la présentation des données directement issues de l'opération. Elles constituent ainsi les éléments de base de nouvelles connaissances, élaborées par l'analyse du fouilleur sur sa propre opération, d'abord directement sur le terrain, ensuite au moment de la rédaction du rapport.

À partir de ces nouvelles connaissances, il est alors possible de publier des études approfondies et des synthèses de plus large portée, qu'elles soient monographiques ou thématiques. Ces synthèses archéologiques, réalisées à partir des rapports de fouille - voire des documents bruts - peuvent être le résultat de recherches réalisées par d'autres que les fouilleurs eux-mêmes, parfois même longtemps après la réalisation de l'opération. Elles peuvent aussi s'appuyer sur un ensemble d'opérations et sur des données issues de recherches non archéologiques. Ces études approfondies répondent chacune à une problématique particulière, différente selon les objectifs poursuivis et selon les méthodes employées pour traiter l'information, sachant que celles-ci évoluent dans le temps non seulement en fonction des données disponibles, mais aussi des régimes d'historicité du moment. C'est bien pourquoi les données sont sans cesse reprises et que leur intégrité, conservation et transmission doivent être garanties. Ces synthèses produisent alors des connaissances de deuxième ordre dont la valeur dépend de celle des données primaires du rapport. Sur la base de ces synthèses, des historiens, géographes, naturalistes, ou autres peuvent à leur tour construire leurs propres récits. Il faut souligner que l'expérience montre que l'utilisation directe des données des rapports de fouille par des chercheurs n'ayant pas la connaissance des critères d'appréciation des données archéologiques peut conduire à des contresens préjudiciables.

À cette étape de l'énoncé de ces quelques rappels, il est pertinent et nécessaire d'identifier, trois natures de production, distinctes et successives dans la production des connaissances en archéologie : la documentation de terrain (vestiges et notes, ultimes témoins du site), le rapport de fouille (première formalisation raisonnée des données primaires issues d'une même opération), et enfin les publications (monographie ou synthèse thématique) reprenant les données de l'opération archéologique, en les confrontant généralement avec des données issues d'autres opérations ou d'autres études.

On aura compris à quel point il est capital, pour l'enrichissement des connaissances et la réalisation de ces publications, que documents de fouille et rapports soient conservés et accessibles (sinon le résultat des fouilles serait perdu), mais aussi qu'ils soient étayés et fiables (sinon les données en seraient scientifiquement inexploitables). Il est également important que les publications fassent explicitement référence à leurs sources, qu'il s'agisse des documentations directement issues du terrain, des rapports d'opération ou des publications ultérieures. Ce que l'on peut nommer la traçabilité des informations utilisées est en effet la garantie de la possibilité d'une analyse critique ultérieure des résultats de la recherche.

Rappelons que, ces dernières décennies, de nombreux bilans, nationaux, régionaux ou thématiques, ont été réalisés le ministère de la culture, le CNRA, les CIRA, les services régionaux ou l'Inrap pour évaluer l'état des connaissances. Des bilans interrégionaux, associant les acteurs institutionnels et scientifiques des régions concernées, tentent de faire le point des connaissances et de tracer des perspectives de recherche. Côté synthèse, l'Inrap a organisé des rencontres 
largement ouvertes $\mathbf{1}$, et édité avec La Découverte une collection de synthèses chronologiques à destination d'un large public. Ces quelques exemples, dont on pourrait allonger la liste, jalonnent les efforts de la communauté des archéologues pour tenter de maitriser l'information et produire de nouvelles connaissances.

Pour en arriver là. Cependant, les questions n'ont pas toujours été posées de cette manière, et cela pour (au moins) deux raisons fondamentales, de cause à effet : d'une part l'évolution de la nature et des objectifs et donc des méthodes de la recherche archéologique, d'autre part la nature et la quantité des données mobilisées pour cette recherche.

Des premières investigations de terrain à nos jours, le chemin fut en effet long et progressif.

Des quelques dégagements réalisés durant la plus haute Antiquité aux travaux des «antiquaires » du XVIII ${ }^{\mathrm{e}}$ siècle, l'activité de terrain qui pourrait être assimilée à de l'archéologie correspondait avant tout à la recherche et à la mise en évidence d'objets emblématiques, simples supports de légitimation du discours des élites ou illustration des légendes populaires (avec appui de «matérialités » diverses - objets, manuscrits, monuments réelles et interprétées, voire créées pour l'occasion). Chaque découverte prenait alors directement le statut de connaissance ${ }^{2}$.

À partir du XIX ${ }^{\mathrm{e}}$ siècle, une approche savante commence à se dégager du politique, du mythologique et du religieux, qui jusqu'alors structuraient le discours historique. On assiste à une prise en compte lente et progressive des réalités matérielles du terrain comme base des raisonnements, et non plus comme simple illustration. C'est notamment le cas avec celle du contexte sédimentaire qui, chez les préhistoriens, devient alors la base d'une démarche chrono-culturelle. Cependant, jusquà la moitié du $\mathrm{xx}^{\mathrm{e}}$ siècle, les données acquises passaient encore très rapidement au statut de «nouvelle connaissance archéologique», sans toujours la distance critique nécessaire, même si les principes de la stratigraphie s'imposaient de plus en plus pour caractériser la valeur des vestiges.

En revanche, la seconde moitié du $\mathrm{xx}^{\mathrm{e}}$ siècle a vu le fort développement des méthodes descriptives et quantitatives critiques, que ce soit pour l'enregistrement des sites dégagés (relevés stratigraphiques et planimétriques de plus en plus affinés) ou celui des vestiges exhumés, d'abord les plus emblématiques puis tous, souvent relevés en « $3 \mathrm{D} »(\mathrm{x}, \mathrm{y}, \mathrm{z})$ à partir de 1950-55. La stratigraphie primaire - qui considérait globalement et du même œil une strate et son contenu - ne suffit en effet bientôt plus et les vestiges d'une même couche sont confrontés entre eux et avec le sédiment qui les englobe.

C'est dans ce contexte que, par exemple, les prises de position méthodologiques de LeroiGourhan au CPF de Strasbourg en 1953 sont symptomatiques : il y énonçait clairement que, pour apprécier la représentativité des vestiges
- en l'occurrence il s'agissait des restes de faune issus du sous-sol et en tirer des connaissances sur l'histoire des hommes, il fallait non seulement les confronter au contexte environnemental et climatique de leur époque, mais évaluer l'érosion due aux attaques physico-chimiques des conditions d'enfouissement, autant que ... celle due à l'activité des fouilleurs : c'était là une exhortation à fouiller de manière fine (il parlait alors de " dissection»), et non à se contenter de recueillir les seuls indices propres à étayer l'hypothèse de départ. De la qualité de la fouille dépendait celle des données recueillies. Donc il fallait changer ces méthodes et les adapter à l'échelle de finesse des nouvelles questions posées par les archéologues. On parlerait aujourd'hui de meilleure résolution!

Entre le mise au jour des données et la production des connaissances, il a fallu depuis lors caractériser explicitement les données en fonction de ces trois filtres : l'époque considérée, l'érosion et les perturbations naturelles, l'intervention des archéologues. Depuis lors, ces derniers ont cherché à améliorer cette étape finale pour tirer le meilleur profit des données rescapées du passé.

Les listes des indices propres à construire de nouvelles connaissances se sont ainsi largement étoffées, des objets manufacturés aux écofacts, des restes humains, animaux et végétaux aux prélèvements les plus variés. Les méthodes de fouille autant que de laboratoire se sont adaptées aux objectifs, de la reconstitution des chronologies à celles de l'occupation de l'espace, de la reconstitution des environnements aux dynamiques sociales des groupes humains. Les données acquises sur le terrain, vestiges comme ensemble de la documentation, sont analysées et critiquées. Les connaissances qui en sont tirées sont argumentées et présentées dans des rapports de plus en plus charpentés, et dont le contenu s'est vu progressivement normalisé.

De la recherche à la sauvegarde. Dans le même temps, progressivement, les opérations archéologiques ne sont plus suscitées que par le seul désir d'obtenir de nouvelles connaissances sur les activités humaines. Les années soixante ont été en effet celles de l'émergence de la prise de conscience des dangers des aménagements monumentaux, urbains et ruraux. Ce qui a conduit les pouvoirs publics, sous la pression de la demande sociale, à prendre une série de mesures réglementaires protégeant le patrimoine soit directement (de la loi sur les secteurs sauvegardés en 1962 à celle sur la protection des collections publiques et des biens culturels du 15 juillet 1980, ou celle sur les ZPPAUP de 1983), soit indirectement (nombreux articles du code de l'urbanisme). Le champ des intérêts, donc celui de la recherche de nouvelles connaissances s'élargit alors considérablement.

Traduction de cette évolution des mentalités, dès 1971, les opérations de sauvetage supplantent en nombre celles qui ne le sont pas, et, à partir de 1981 le nombre des autorisations de fouilles délivrées à des "professionnels et assimilés » $(51,5 \%=$ CNRS, université, sous-direction de l'archéologie ${ }^{\mathbf{5}}$, musées, étudiants, collectivités,
Demoule et Stiegler

2 Schnapp (A.) 1993

3 De Beaune (S.) 2010 
étrangers) est supérieur à celui de celles délivrées à des « amateurs » $(48,5 \%=$ autres, enseignants non-universitaires, sans profession, inconnu). Cette augmentation, signe de l'accroissement des professionnels et de celui des opérations de sauvetages, fait que les données nouvelles affluent de plus en plus, les étagères se remplissent de rapports tandis que les dépôts, plus ou moins pérennes, se multiplient. Cette mobilisation de la communauté archéologique se manifeste également par la tenue, en 1981, d'Assises nationales de l'archéologie et par l'émergence d'un périodique d'un genre nouveau : Les nouvelles de l'archéologie.

De leur côté, le ministère de la culture (créé en 1959) et le CSRA (créé en 1964) s'emparent de la situation nouvelle en tentant d'organiser les interventions, d'abord en en "programmant» le calendrier, puis en regroupant les fouilles par «grands thèmes » chrono-culturels. Cependant, par une définition très circulaire, ce projet de rationalisation scientifique ne concerne que les opérations «programmées » (fouilles et sauvetages du même nom) et non celles, de plus en plus nombreuses et réalisées dans l'urgence du calendrier. Cet aspects était explicitement affiché par le représentant du ministre, Jean Gazagnes, devant le CSRA en février 1975 : « les thèmes de recherches ne peuvent être envisagés que dans le cadre des fouilles traditionnelles, dites «programmées » sur l'autorisation desquelles le conseil supérieur est appelé à donner son avis ; les fouilles de sauvetage ne sauraient bien sûr être soumises à de telles directives puisqu'elles doivent nécessairement être menées d'urgence sur tout gisement archéologique menacé ou en cours de destruction ». Un nouveau filtre, sélectif et de nature administrative, destiné notamment à laisser plus de latitude aux directeurs de circonscription, mais limitant de fait l'ampleur des connaissances s'instaure alors avec l'exclusion des données issues des opérations d'urgence.

La réflexion conjointe du ministère et du CSRA aboutit néanmoins, en 1980 et après des années de débats et plusieurs ébauches de ces «thèmes de recherches », à la première «programmation archéologique» officielle ${ }^{6}$.

Il en résulte un élan et un saut qualitatif important car l'augmentation des opérations «programmés » - sauvetages et fouilles - et l'afflux des données nouvelles qui en résulte, amène la communauté archéologique à tracer de nouvelles perspectives. Les connaissances nouvelles dessinent un nouveau paysage archéologique de la France, paysage qui prend corps notamment avec les diverses manifestations de l' « année de l'archéologie », décrétée en 1989: exposition sur l'apport des méthodes de laboratoires (Les mystères de l'archéologie ${ }^{7}$ ), exposition au Grand Palais et édition de l'Archéologie de la France ${ }^{\mathbf{8}}$, labélisation de chantiers, etc.

Le CSRA doit même modifier les périmètres de ses «programmes» pour les adapter à cet afflux de nouvelles connaissances. Le rapport quinquennal, édité pour la première fois par le ministère en 1989, fait en effet largement état de ce profond renouvellement des connaissances et des pratiques institutionnelles en archéologie. Pour autant, pour établir ses propositions de « programmes », le CSRA n'a pas toujours accès aux résultats des opérations «non programmées», dont l'ampleur et l'importance grandit. Elles sont de plus devenues majoritaires et largement mieux dotées que les opérations de «fouilles programmées » du fait des évolutions de la réglementation, notamment avec celle sur les grands travaux et les études d'impact. Cette situation fait que la «connaissance officielle» est celle retenue par un tamis à mailles de plus en plus larges, laissant passer une part de plus en plus importante de l'information. Il faudra la démission du CSRA, en 1991, pour que soit enfin ouvert, avec la création du CNRA et des CIRA en 1994, le chemin vers une prise en compte de l'ensemble des opérations, mêmes celles appelées «préventives» depuis qu'elles sont organisées en amont des aménagements et non plus dans l'urgence de leurs phases de réalisation.

Par ailleurs, les chercheurs eux-mêmes n'ont en général que difficilement accès aux données et vestiges, ou même aux rapports de fouille, quels qu'ils soient. Or l'augmentation réelle et non biaisée des connaissances n'est possible que par un libre accès aux données du terrain, dont les rapports sont un témoignage essentiel. Le ministère, conscient du problème, tente bien, au début des années quatre-vingt-dix ${ }^{9}$, de rappeler l'obligation légale de communication, mais les habitudes de rétention de l'information persistent souvent.

En cette fin de vingtième siècle, on peut donc considérer qu'un filtre important s'impose entre les données du terrain et la production des connaissances : celui de l'accessibilité aux données et rapports. Ce filtre n'est pas nouveau, mais il prend une nouvelle dimension avec l'augmentation de la demande, correspondant à un renouvellement du besoin de données pour la recherche. Quoi qu'il en soit, si le filtre de la qualité des fouilles était du seul ressort des fouilleurs, celui de l'accessibilité dépend de deux autres facteurs supplémentaires : les conditions matérielles de conservation et de consultation, et les contraintes réglementaires! Certaines forces d'inertie, tant des responsables de fouilles que de l'administration en région, peuvent également être à l'origine d'un accès difficile aux données...
4. PV de la session plénière du CSRA de février 1972.

5 Les personnels de l'Afan sont alors comptabilisés par le ministère de la Culture avec ceux des « circonscriptions » sous la rubrique «SDA ». 6 Programmation de la recherche archéologique de terrain en France, CSRA et Ministère de la culture, 1981, 104 p.
7 Collectif, 1990 : Les mystères de l'archéologie, les sciences à la recherche du passé, PUL et CNMHS, $287 \mathrm{p}$. 8 Ouvrage collectif 9 Braize (F.) 1990 L'amélioration de l'accès des citoyens aux documents administratifs du secteur culturel, rapport au directeur de cabinet,ministère de la culture; Les rapports de fouille : notion, communicabilité et évolution, Séminaire du 15 novembre 1990, et «circulaire DFS » en 1993 
Des données et informations nouvelles à cumuler et confronter. Où en sommes-nous en ce début de $\mathrm{XXI}^{\mathrm{e}}$ siècle? Quelles que soient les modifications récentes des modalités des pratiques ou les circonstances des opérations, un des fondements majeurs de l'archéologie reste de caractériser les évènements et les chronologies, les dynamiques spatiales et les caractérisations fonctionnelles, pour élaborer un discours - original et possédant ses propres modalités par rapport à une histoire plus textuelle - sur l'histoire des hommes en société. Qu'elles modifient radicalement les connaissances, ou qu'elles en confortent d'anciennes, les nouvelles données contribuent toutes à l'enrichissement $\mathrm{du}$ fonds exploitable. Si les connaissances nouvelles servent le plus souvent à affiner le degré de résolution des anciennes, elles concourent aussi à en renouveler l'approche, l'accroissement de leur quantité et une meilleure précision de leur répartition provoquant un effet de seuil qualitatif.

Des domaines de connaissance élargis et enrichis. Avec l'élargissement de l'éventail des champs disciplinaires convoqués dans le cadre de la recherche archéologique, il s'agit aussi bien de restituer les rapports dans et entre groupes humains qu'avec leur environnement, ce qui implique de distinguer ce qui, dans l'environnement, influe sur le comportement des hommes et ce qui, dans l'action des hommes, agit sur l'environnement, proche ou lointain, à court ou long terme.

De plus, soulignons que, depuis au moins une vingtaine d'années, les périodes les plus récentes de l'histoire (époque dite «moderne et contemporaine») bénéficient enfin, et de manière moins exceptionnelle, de l'attention des archéologues. Cela tient à deux éléments, l'un théorique - la prise d'autonomie de la démarche archéologique dans le champ des disciplines de l'histoire fait qu'aucune période n'en est exclue -, l'autre pratique -

le développement d'une archéologie préventive systématique, ce qui a ouvert la possibilité de la prise en compte des niveaux stratigraphiques supérieurs et affleurants, que sont les traces imprimées dans les sites et les paysages. Cette approche archéologique de périodes traditionnellement abordées par les textes et l'histoire de l'art, permet aussi de mieux caractériser son originalité et sa pertinence dans la construction du discours historique.

Enfin, l'archéologie s'appuie désormais sur une approche globale et transversale, donc par essence inter-disciplinaire. Cela implique d'exploiter toutes les sources, sans en hiérarchiser a priori la valeur, en fonction d'objectifs qui permettent, au départ, de définir à chaque fois une perspective commune de recherche archéologique : histoire technique, économique, sociale ; dynamiques régionales ou interrégionales; échelles de synchronie ou de diachronie, etc.

Aujourd'hui, cela signifie qu'il n'y a plus de « discipline annexe» fournissant des données brutes, élaborées en marge, dans un cadre distinct et spécifique, mais bien des «disciplines contributives», des concours méthodologiques croisés et intégrés dans des problématiques autour de l'homme en société. Il s'agit donc d'une batterie de nouveaux filtres, cette fois-ci optimisants, entre la production des données et leur exploitation pour de nouvelles connaissances : la caractérisation et la dénomination des faits issus d'approches connexes et diversifiées. La caractérisation et la dénomination des vestiges recueillis et des faits observés cumule maintenant plusieurs items : lieu, matière, chronologie, datation, fonction, etc. Aucun n'est intangible et tous peuvent évoluer, au moins dans leur formulation ou leur précision. Ils s'organisent généralement entre eux en systèmes typologiques qui, à leur tour, contribuent à façonner les raisonnements. Il faut désormais organiser les conditions théoriques et pratiques d'une cohésion qui en permette la confrontation.

\section{Des outils méthodologiques (parmi d'autres) à toujours mieux définir. \\ L'apport de la modélisation}

Depuis longtemps les interprétations des données produisent de nouvelles connaissances en s'appuyant sur leur confrontation avec le contexte, spatial ou stratigraphique, synchro ou diachronique, et la comparaison avec des faits et des données de même nature connus par ailleurs. Elles ne se font généralement pas à la seule échelle du site.

Cependant, depuis le dernier quart du $\mathrm{Xx}^{\mathrm{e}}$ siècle, les archéologues proposent fréquemment soit des résultats explicitement adossées à des modélisations, soit des modèles descriptifs ou interprétatifs sur la base de leurs découvertes. Ces modèles permettent de donner une autre image des séries de données prises en compte, et de les confronter à des images similaires. En effet, élaborés statistiquement en fonction d'une hypothèse, ou à partir des faits enregistrés et des données élémentaires, ils s'avèrent bien souvent indispensables à la stimulation de la recherche et à l'ouverture de nouvelles voies d'investigation. Cette tendance s'est renforcée avec les outils de géo-référencement. Pour autant, ces modèles ne sont légitimement aptes à proposer une explication des combinaisons des faits qu'ils retiennent dans ceux enregistrés par l'archéologie, qu'à la condition que les chercheurs qui élaborent ces modèles théoriques n'aient de cesse de les remettre en cause à chaque découverte d'ensemble de faits nouveaux. Ce qui ne manque justement pas de se produire avec l'augmentation des opérations de terrain et l'afflux des données nouvelles. Théoriquement, cela implique la nécessité de confronter des données ayant été obtenues avec le même degré d'investigation et d'élaborer des problématiques croisées en identifiant des échelles compatibles d'analyse et de restitution. Pratiquement, cela impose un enregistrement des données ainsi qu'un travail de vocabulaire et d'indexation qui en permette le repérage, l'usage et l'exploitation systématique.

C'est bien pourquoi il importe non seulement d'enregistrer rigoureusement toutes les données de terrain, indépendamment de ce qui est nécessaire à la validation du modèle ayant permis d'établir la problématique de la fouille par exemple mais de les présenter, dans les rapports ${ }^{\mathbf{1 0}}$, de manière systématique et le plus accessible possible à d'autres type de questionnement. 


\section{Lévaluation de la représentativité}

La question de la représentativité s'est posée dès que les données ont été évaluées d'un œil critique (cf. les analyses de Leroi-Gourhan citées plus haut) ou ont été quantifiées : ce fut le cas dans les années soixante avec les discussions sur la valeur des diagrammes cumulatifs et des listes-types de Denise de Sonneville-Bordes et Jean Perrot ; ou encore avec la critique des interprétations fonctionnelles d'un site partiellement fouillé ou d'une série lithique tronquée... Sans compter les différences observées en comparant les séries issues de sites selon que les sédiments sont ou non tamisés.

Plus récemment, que se soit avec les fouilles de la vallée de l'Aisne dirigées par Bohumil Soudsky dans les années soixante-dix ou vingt ans plus tard avec la question des pourcentages d'ouverture à réaliser dans les opérations de diagnostic, la question de la valeur de l'échantillonnage a été prépondérante. Avec les outils actuels de géoréférencement, il devient possible de travailler rapidement à différentes échelles, et la question de la représentativité, donc de la qualification des données à prendre en compte pour aller vers de nouvelles connaissances, se pose dans de nouveaux termes ${ }^{\mathbf{1 1}}$.

Aujourd'hui pour demain? Au-delà d'une proposition banale et rebattue, cette question reflète une réalité bien concrète. La lenteur des processus réglementaires (que de décennies de luttes et de paliers pour arriver à la loi de 1941 et de celle-ci à la loi sur l'archéologie préventive soixante ans plus tard), incite à penser que les réflexions menées aujourd'hui ne seront pas appliquées avant quelques années. Cependant, il nous faut faire vite car chacun sait à quel rythme s'érodent désormais les terrains potentiellement fournisseurs de données.

Après une phase de travail isolé (les savants du XIX ${ }^{e}$ et début $\mathrm{XX}^{\mathrm{e}}$ ), de travail en équipe (deuxième moitié $\left.d u 20^{\mathrm{e}}\right)$, puis en réseaux, il s'agit prolonger la recherche vers le travail avec des outils documentaires partagés (techniques, mais aussi institutionnels donc à dimension politique). En effet, à chacune de ces étapes, correspond un degré de conservation des données primaires. Nous sommes ainsi passé du simple dépôt lapidaire passif au dépôt de fouille actif, organisé avec un centre de conservation et d'étude ${ }^{\mathbf{1 2}}$ et de documentation mettant à disposition les travaux, analyses et rapports, voire les publications qui sont en lien avec les vestiges conservés. La question des conditions d'accès aux connaissances nouvelles issues des opérations concerne désormais aussi bien les mobiliers et documentations primaires, que les rapports, aussi bien les opérations programmées que les préventives, autant celles réalisées par le secteur public, que par les opérateurs privés.

Il s'agit également de mettre en place une traçabilité des connaissances : citer ses sources et indiquer les références des méta-données, pour le papier comme pour le numérique. Il ne faut pour cela ni usine à gaz, ni «simple bon sens», mais des outils performants de gestion et de stockage et d'interrogation, sans oublier un travail en réseaux documentaires. En effet, quels que soient les outils techniques élaborés, ils ne pourront fonctionner et évoluer sans le concours des chercheurs qui s'en servent ni celui des praticiens de l'archivage, de la documentation et de la conservation.

Cette nouvelle étape implique un nouveau chantier, déjà commencé et à élargir en tenant compte des éléments ci-dessus, impliquant toutes les catégories d'acteurs concernés, tant techniquement et scientifiquement qu'institutionnellement. Rappelons que la loi de décentralisation du 7 janvier 1983 déclare le territoire "patrimoine commun de la Nation». Il est donc légitime, pour une nouvelle ambition dans la construction des connaissances ${ }^{\mathbf{1 3}}$, de demander la nationalisation des données en archéologie : c'est-à-dire aussi bien la propriété publique des vestiges et documentations de toutes natures issus des opérations archéologiques, quel qu'en soit le statut juridique, que l'organisation du libre accès aux documents, vestiges et rapports d'opération.

Collectif, 1990 : Archéologie de la France, RMN, coll. Champs Flammarion, $509 \mathrm{p}$.

Archambault de Beaune S. (Dir.), 2010 : Écrire le passé, CNRS éditions, $425 \mathrm{P}$.

Braize F., 199o, L'amélioration de l'accès des citoyens aux documents administratifs du secteur culturel, rapport au directeur de cabinet, ministère de la culture ; Les rapports de fouille : notion, communicabilité et évolution, Séminaire du 15 novembre 1990, et « circulaire DFS» en 1993.

Demoule J.-P. (DIR.), 2007: L'archéologie préventive dans le monde, apports de l'archéologie préventive à la connaissance du passé, La Découverte, $286 \mathrm{p}$.

Demoule J.-P. et Stiegler B. (Dir.), 2008 : L'avenir du passé, modernité de l'archéologie, La Découverte, $250 \mathrm{p}$.

Deyber-Persignat D. (DIR.), 2000 : Le dépôt archéologique, Conservation et gestion pour un projet scientifique et culturel, Assises nationales de la conservation archéologique, Bourges 26-28 novembre 1998, éditions de la ville de Bourges.

COLleCtif, 1990 : Les mystères de l'archéologie, les sciences à la recherche du passé, PUL et CNMHS, $287 \mathrm{p}$.

Magnan A., Aubin G., RutschKowsky M., BeL N., 2007 : L'application des textes relatifs au mobilier archéologique, rapport $\mathrm{n}^{\circ} 2077-05$, ministère de la culture et de la communication, IGAC, mars 2007, 77 p.

Soulier PH., 2003 : «Soixante ans d'organisation de l'archéologie en France : de la loi de 1910 à celle de 2001 ", in PoIrrier PH. et VADElorge L. (DIR.), Pour une histoire des politiques du patrimoine, Comité d'histoire du ministère de la Culture, fondation MSH, Paris, p. 429-450.

SOUlier PH., 2010 : «Archéologie : écriture de l'histoire et réglementation des fouilles en France», in DE BEAUNE S. (DIR.), Ecrire le passé, CNRS Editions, p. 29-46.

SOULIER PH. (DIR.), 2011 : Le rapport de fouille archéologique: réglementation, conservation, diffusion, Travaux de la Maison René-Ginouvès, 11, De Boccard, $190 \mathrm{p}$.

Précis méthodologique pour la création des Centres de Conservation et d'Étude (CCE), 2008 : ministère de la culture et de la communication, DAPA, Sdarchetis, $10 \mathrm{p}$.

PAPINOT J.-Cl. (avec la coll. de G. Verron), $1998:$ La conservation $d u$ mobilier archéologique, rapport à monsieur le directeur de l'architecture et du patrimoine, I Rapport de synthèse, 35 p.

SChNapp A., 1993 : La conquête du passé, aux origines de l'archéologie, éd. Carré, 384 p.

11 Voir à ce sujet le thème du prochain colloque de Châlonsen-Champagne. 12 Papinot et Verron 1993, Deybert-Pressignat dir. 200o, Magnant et coll. 2007, Précis méthodologique, 2008 13 Magnant et coll. 2007. 